\title{
BERNARD KLARES
}

\section{Problème de la stabilité structurelle des champs de vecteurs holomorphes sur certaines surfaces algébriques}

Mémoires de la S. M. F., tome 38 (1974), p. 73-78

<http://www.numdam.org/item?id=MSMF_1974_38_73_0>

CC Mémoires de la S. M. F., 1974, tous droits réservés.

L'accès aux archives de la revue « Mémoires de la S. M. F. » (http://smf. emath.fr/Publications/Memoires/Presentation.html) implique l'accord avec les conditions générales d'utilisation (http://www.numdam.org/conditions). Toute utilisation commerciale ou impression systématique est constitutive d'une infraction pénale. Toute copie ou impression de ce fichier doit contenir la présente mention de copyright.

\section{Numdam}


PROBLEME DE LA STABILITE STRUCTURELLE DES CHAMPS

DE VECTEURS HOLOMOR PHES SUR CERTAINES SURFACES ALGEBRIQUES

par Bernard KLARES

\section{I - Problème de la stabilité structurelle}

Soient $M$ une variété analytique complexe, compacte de dimension $\mathrm{n}$ et $\vartheta(\mathrm{M})$ l'ensemble des champs de vecteurs holomorphes sur M.

$\vartheta(M)$ est un espace vectoriel de dimension finie, que l'on supposera muni d'une norme qui en fait un espace de Banach.

\section{DEFINITIONS:}

- $V \in \mathcal{V}(M)$ et $W \in V(M)$ sont dits analytiquement équivalents, s'il existe un automor phisme analytique de $M$, qui transforme $V$ en $W$.

$-V \in V(M)$ et $V^{\prime} \in V(M)$ sont dits topologiquement équivalents, s'il existe un homéomorphisme de $M$ sur $M$, qui envoie les trajectoires de $V$ sur celles de $V^{\prime}$.

- $V \in \mathscr{F}(M)$ est dit structurellement stable, s'il existe un ouvert $U_{M}(V)$ contenant $V$ et tel que tout $V^{\prime}$ de $U_{M}(V)$ soit topologiquement équivalent à $V$.

\section{Problème de la stabilité structurelle}

L'ensemble des champs de vecteurs holomorphes structurellement stables sur $M$ est un ouvert de $\mathscr{V}(\mathrm{M})$. Est-il partout dense ou non dans $\mathscr{V}(\mathrm{M})$ ? On va résoudre ce problème dans le cas où $M$ est obtenue à partir de $\mathbb{P}_{1}(\mathbb{C}) \times \mathbb{P}_{1}(\mathbb{C})$ par éclatements successifs.

II - Recherche des champs de vecteurs holomorphes sur les surfaces considérées.

On va d'abord étudier le problème de l'éclatement.

1. Champs de vecteurs holomorphes et éclatement

On considère : $M$ une variété analytique complexe, de dimension supérieure ou égale à deux, $K$ une sous-variété de codimension supérieure ou égale à deux, M' la variété obtenue par éclatement de Hopf le long de $K, V_{K}(M)$ I'ensemble des champs de vecteurs holomorphes sur M, s'annulant sur K et $\vartheta\left(M^{\prime}\right)$ l'espace vec- 
toriel des champs de vecteurs holomorphes sur $M^{\prime}$. On a :

PROPOSITION 1. $\vartheta_{\mathrm{K}}(\mathrm{M})$ et $v\left(M^{\prime}\right)$ sont deux espaces vectoriels isomorphes.

Démonstration : On va faire la démonstration dans le cas où dim $M=2$ et $K$ est un point $\mathrm{m}$. Soient $(\mathrm{x}, \mathrm{y})$ des coordonnées locales au voisinage de $\mathrm{m}$, telles que $\mathrm{m}$ corresponde à $\mathrm{x}=0$ et $\mathrm{y}=0$. Soit $\mathrm{V} \in \boldsymbol{V}(\mathrm{M})$ tel que $\mathrm{V}=\mathrm{P}(\mathrm{x}, \mathrm{y}) \partial / \partial \mathrm{x}+\mathrm{Q}(\mathrm{x}, \mathrm{y}) \partial / \partial \mathrm{y}$ au voisinage de $\mathrm{m}$. Si $\pi: M^{\prime} \rightarrow M$ est la projection associée à l'éclatement, et si $\mathrm{m}^{\prime} \in \pi^{-1}(\mathrm{~m})$ $i l$ existe des coordonnées locales au voisinage de $m^{\prime}:(u, v)$ telles que $\mathrm{u}=\mathrm{x} \quad \mathrm{v}=\mathrm{y} / \mathrm{x} \quad\left(\mathrm{ou} \quad \mathrm{u}^{\prime}=\mathrm{x} / \mathrm{y} \quad \mathrm{v}^{\prime}=\mathrm{y}\right.$ suivant la position de $\mathrm{m}^{\prime}$ sur $\pi^{-1}(\mathrm{~m})$ ) Alors $V$ définit un champ de vecteurs $\tilde{V}$ qui s'écrit au voisinage de $m^{\prime}$ : $\tilde{\mathrm{V}}=\tilde{\mathrm{P}}(\mathrm{u}, \mathrm{v}) \partial / \partial \mathrm{u}+\tilde{\mathrm{Q}}(\mathrm{u}, \mathrm{v}) \partial / \partial \mathrm{v}$ avec : $\tilde{\mathrm{P}}(\mathrm{u}, \mathrm{v})=\mathrm{P}(\mathrm{u}, \mathrm{uv}) \quad \tilde{\mathrm{Q}}(\mathrm{u}, \mathrm{v})=-(\mathrm{v} / \mathrm{u}) \mathrm{P}(\mathrm{u}, \mathrm{uv})+(1 / \mathrm{u}) \mathrm{Q}(\mathrm{u}, \mathrm{uv})$

Pour que $V$ soit holomorphe il faut et il suffit que $P(0,0)=0$ et $Q(0,0)=0$ donc que $V$ s'annule en $m$.

Réciproquement à tout champ de vecteurs holomorphe sur $M^{\prime}$ on peut associer (par la projection $\pi$ ) un champ de vecteurs holomorphe sur M' qui s'annule en m.

\section{Conséquences}

- Si on éclate $\mathbb{P}_{2}(\mathbb{C})$ en quatre points formant un repère, sur la surface obtenue il n'y a qu'un seul champ de vecteurs holomorphe : le champ nul. En effet il n'existe aucun champ de vecteurs holomorphe sur $\mathbb{P}_{2}(\mathbb{C})$, non nul, qui admette les quatre points considérés comme points singuliers $\mathfrak{v}_{\mathrm{K}}\left(\mathbb{P}_{2}(\mathbb{C})\right)$ est alors réduit à 0 donc $\vartheta\left(M^{\top}\right)$ aussi, plus généralement :

- Si M , sous-variété de $\mathbb{P}_{N}(\mathbb{C})$, contient $N+2$ points formant un repère, et si l'on éclate $M$ en $N+2$ points, sur la variété obtenue, il n'y a qu'un seul champ de vecteurs holomorphe : le champ nul.

En effet d'après la proposition 1 il suffit de chercher les champs de vecteurs holomorphes sur $M$ qui s'annulent en ces $N+2$ points. Comme tout champ de vecteurs holomorphe sur $M$ qui possède un point singulier, au moins, se prolonge en un champ de vecteurs de $\mathbb{P}_{\mathrm{N}}(\mathbb{C})$, ([2]) et qu'il n'existe aucun champ de vecteurs holomorphe de $\mathbb{P}_{N}(\mathbb{C})$ non nul qui possède ces $N+2$ points comme points singuliers, le résultat s'en déduit immédiatement.

- Cette proposition montre, en outre, qu'il y a de "nombreuses" surfaces compactes ne possédant que le champ nul, comme champ de vecteurs holomorphe plus précisément : Carrel, Howard et Kosniowski ont déterminé dans un récent article, toutes 
les surfaces compactes admettant un champ de vecteurs holomorphe non trivial et possédant des singularités [1].

- On va maintenant appliquer ces résultats aux surfaces considérées. On peut résumer cette étude par

PROPOSITION 2. Il existe un ouvert $\theta(M)$ partout dense dans $v(M)$, une carte $U$ et des coordonnées locales $(x, y)$ dans $U$, tels que tout champ de vecteurs $V$ de $\theta(M)$ soit analytiquement équivalent à un champ de vecteurs $W$ de $\theta(M)$ qui s'écrit dans $U$ :

$$
\begin{aligned}
& W=\mathrm{P}(\mathrm{x}, \mathrm{y}) \partial / \partial \mathrm{x}+\mathrm{Q}(\mathrm{x}, \mathrm{y}) \partial / \partial \mathrm{y} \text { avec } \\
& \alpha\left\{\begin{array} { l } 
{ \mathrm { P } ( \mathrm { x } , \mathrm { y } ) = \mathrm { ax } } \\
{ \mathrm { Q } ( \mathrm { x } , \mathrm { y } ) = \mathrm { by } }
\end{array} \quad \boldsymbol { \beta } \left\{\begin{array} { l } 
{ \mathrm { P } ( \mathrm { x } , \mathrm { y } ) = \mathrm { ax } ^ { 2 } } \\
{ \mathrm { Q } ( \mathrm { x } , \mathrm { y } ) = \mathrm { by } ^ { 2 } }
\end{array} \quad \text { 目 } \left\{\begin{array}{l}
\mathrm{P}(\mathrm{x}, \mathrm{y})=0 \\
\mathrm{Q}(\mathrm{x}, \mathrm{y})=0
\end{array}\right.\right.\right.
\end{aligned}
$$

Les différents cas obtenus dépendent bien sûr de la position des points où l'on fait successivement, les éclatements.

\section{III - Stabilité structurelle}

Tous les champs considérés dans cette partie appartiendront à $\theta(\mathrm{M})$ (proposition 2).

1. Cas de $\mathbb{P}_{1}(\mathbb{C}) \times \mathbb{P}_{1}(\mathfrak{c})=\mathbb{M}_{0}$

On est dans le cas de $\propto$ de la proposition 2) avec $a \neq 0$ et $b \neq 0$ et

$a \neq b$. Tout champ $V$ de $\theta\left(M_{0}\right)$ est donc analytiquement équivalent à un champ $W$. qui a pour composantes dans $U:\left\{\begin{array}{l}P(x, y)=a x \\ Q(x, y)=b y\end{array}\right.$

Etudions les trajectoires de W - W possède quatre points singuliers :

$\mathrm{m}(\mathrm{x}=0, \mathrm{y}=0), \mathrm{m}_{1}\left(\mathrm{x}_{1}=0, \mathrm{y}_{1}=0\right)$ avec $\mathrm{x}_{1}=1 / \mathrm{x}, \mathrm{y}_{1}=\mathrm{y}, \mathrm{m}_{2}\left(\mathrm{x}_{2}=0, \mathrm{y}_{2}=0\right)$

avec $\mathrm{x}_{2}=\mathrm{x}, \mathrm{y}_{2}=1 / \mathrm{y}$ et $\mathrm{m}_{3}\left(\mathrm{x}_{3}=0, \mathrm{y}_{3}=0\right)$ avec $\mathrm{x}_{3}=1 / \mathrm{x}, \mathrm{y}_{3}=1 / \mathrm{y}$.

Il y a quatre trajectoires homéomorphes à des cylindres : $\left\{\begin{array}{l}\mathrm{x}=0 \text { ou } \mathrm{y}=0 \\ \text { ou } \mathrm{x}_{3}=0 \text { ou } \mathrm{y}_{3}=0\end{array}\right.$

et dans le cas où $\operatorname{Im}(-a / b) \neq 0$ toutes les autres trajectoires sont homéomorphes à des plans admettant le paramétrage suivant :

$$
\text { (1) }\left\{\begin{array}{l}
\varphi=\frac{1}{\operatorname{Imd}} \log \frac{\mathrm{r} \cdot \mathrm{s} \cdot .^{\operatorname{Red}}}{|\mathrm{k}|} \\
\theta=\operatorname{Argk}-\frac{1}{\operatorname{Imd}} \log \frac{\mathrm{r} \cdot \mathrm{s}}{|\mathrm{k}|^{\operatorname{Red}}}|\mathrm{d}|^{2}
\end{array}\right.
$$

où : $\mathrm{d}=-\mathrm{a} / \mathrm{b}, \mathrm{x}=r \mathrm{e}^{i \theta} \mathrm{y}=\mathrm{se}$ 
Ces trajectoires homéomorphes à des plans "s'enroulent" autour des trajectoires homéomorphes à des cylindres puisque lorsque $r$ ou $s$ tendent vers zéro, $\varphi$ et $\theta$ tendent vers l'infini.

Soit $\theta^{\prime}(M)$ l'ensemble des champs de vecteurs de $\theta(M)$ analytiquement équivalents aux champs $W$ qui vérifient $\operatorname{Im}(a / b) \neq 0$. Nous allons montrer que tout champ de vecteurs de $\theta^{\prime}(M)$ est structurellement stable et par conséquent' le problème sera résolu puisque $\theta^{\prime}(M)$ est un ouvert partout dense de $v(M)$.

Soit $V \in \theta^{\prime}(M)$ et $V_{M}(V)$ l'ensemble des champs de vecteurs de $\theta^{\prime}(M)$ analytiquement équivalents aux champs $W^{\prime}$ qui vérifient $\operatorname{Im} d$. Im $d^{\prime}>0\left(d^{\prime}=-a^{\prime} / b^{\prime}\right)$. Montrons que $V$ et $V^{\prime}$ de $V_{M}(V)$ sont topologiquement équivalents. $V$ est analytiquement équivalent à $W$ et $V^{\prime}$ à $W^{\prime}$, il suffit donc de montrer que $W^{\text {et }} W^{\prime}$ sont topologiquement équivalents. Pour cela on va construire un homéomorphisme $h$ qui envoie les trajectoires de $W$ sur celles de $W^{\prime}$ :

- Ie paramètrage (1) montre que toute trajectoire de W (et de $W^{\prime}$ ) coupe le tore $\boldsymbol{b}: \mathrm{r}=1$ et $\mathrm{s}=1$ en un point et un seul. Soit $\mathrm{h} / \mathrm{b}=\mathrm{Id}$ ce qui donne la correspondance entre les trajectoires de $W$ et celles de $W^{\prime}$. Il reste à montrer qu'il existe une correspondance entre les points des trajectoires de $W$ et celles de $W^{\prime}$, qui définit une application se prolongeant en un homéomorphisme de $\mathbb{P}_{1}(\mathbb{C}) \times \mathbb{P}_{1}(\mathbb{C})$ dans lui-même.

Associons au point $(r, s)$ de la trajectoire de $W$, le point $\left(r^{\prime}, s^{\prime}\right)$ de 'la trajectoire de $W^{\prime}$ (déterminé par la définition de $h$ sur $\mathbf{r}$ ) tel que :

$$
r^{\prime}=r^{\frac{\text { Imd }}{\text { Imd }}} \quad s^{\prime}=s^{\frac{\left.|d|^{2} \cdot d^{\prime}\right|^{2} \text { Imd }}{\mid d^{\prime}}}
$$

On a alors $\theta^{\prime}=f_{1}(\theta, r) \quad \varphi^{\prime}=g_{1}(\varphi, s) \quad\left(\right.$ avec $h(x, y)=\left(x^{\prime}, y^{\prime}\right)$ et des notations évidentes).

Ceci montre que l'application ainsi construite se prolonge sur les trajectoires particulières (lorsque $r$ tend vers zéro ou $\mathbf{s}$ tend vers zéro) d'une façon continue, de même qu'aux points singuliers qui sont laissés invariants (Imd . Imd'>0).

$\mathrm{h}$ ainsi contruit est un homéomorphisme qui envoie les trajectoires de $W$ sur celles de $W^{\prime}$ et le problème est résolu.

2. Cas où $M$ est obtenue à partir de $\mathbb{P}_{1}(\mathfrak{C}) \times \mathbb{P}_{1}(\mathbb{C})$ par éclatement en un point

on est encore dans le cas $\alpha\left\{\begin{array}{l}a \neq 0 \text { et } b \neq 0 \text { de la proposition 2. On } \\ \text { et } a \neq b\end{array}\right.$ procède de manière analogue à la précédente :

on considère $\theta^{\prime}\left(M_{1}\right)$ l'ensemble des champs de vecteurs de $\theta\left(M_{1}\right)$ analytique- 
ment équivalents aux champs $W$ qui vérifient: $\operatorname{Im}(-a / b) \neq 0$ et on montre que tout champ $V$ de $\theta^{\prime}\left(\mathbb{M}_{1}\right)$ est structurellement stable.

Si $V \in \theta^{\prime}\left(M_{1}\right)$ on appelle $U_{\mathbb{M}_{1}}(V)$ l'ouvert contenant $V$ défini comme suit :

$\mathrm{U}_{M_{1}}(V)$ est l'ensemble des champs de vecteurs holomorphes de $\theta^{\prime}\left(M_{1}\right)$ analytiquement équivalents aux champs $W^{\prime}$ tels que $: \operatorname{Im}(\mathrm{a} / \mathrm{b}) \operatorname{Im}\left(\mathrm{a}^{\prime} / \mathrm{b}^{\prime}\right)>0$. Montrons que $V$ et $V^{\prime}$ de $U_{\mathbb{M}_{1}}(V)$ sont topologiquement équivalents. Pour cela il suffit de le montrer pour $W$ et $W^{\prime}$.

Remarquons que le point $m$ où l'on éclate correspond à $x=0$ et $y=0$ et que $\mathrm{x}$ et $\mathrm{y}$ sont des coordonnées locales "habituelles" de $\mathbb{P}_{1}(\mathbb{C}) \times \mathbb{P}_{1}(\mathbb{C})$ (les changements de cartes sont les mêmes que ceux définis précédemment). On procède de la même façon que dans le cas (1). Toute trajectoire non homéomor phe à un cylindre coupe $\boldsymbol{l}$ en un point et un seul et on pose $\mathrm{h} / \boldsymbol{h}=\mathrm{Id}$.

Il faut montrer que l'on peut choisir la correspondance des points sur les trajectoires de $W^{\text {et }} W^{\prime}$, de façon que l'application ainsi définie se prolonge en un homéomorphisme de $M_{1}$. Pour cela on va modifier la correspondance définie précédemment au voisinage de $\mathrm{m}$, plus précisément dans $\mathrm{E}=\{\mathrm{p}(\mathrm{x}, \mathrm{y})|| \mathrm{x} \mid \leqslant 1$ et $|\mathrm{y}| \leqslant 1\}$ :$$
\text { Soit } E_{1}=\{(x, y) \mid r \leqslant s \leqslant 1\} \quad x=r e^{i \theta} \quad y=s e^{i \varphi}
$$$$
\mathrm{E}_{2}=\{(\mathrm{x}, \mathrm{y}) \mid \mathrm{s} \leqslant \mathrm{r} \leqslant 1\}
$$

Dans $E_{1}$ au point $(r, s)$ de la trajectoire de $W$ on associe le point $\left(r^{\prime}, s^{\prime}\right)$ de la trajectoire de $W^{\prime}$ tel que :

$$
\left\{\begin{array}{l}
\vdots \\
r^{\prime}=r^{\frac{\alpha}{\alpha^{\prime}}} \\
s^{\prime}=s^{\frac{|d|^{2} \text { Imd' }}{\left|d^{\prime}\right|^{2} \operatorname{Imd}}} \quad r^{\frac{\alpha}{\alpha^{\prime}}-\frac{|d|^{2} \text { Imd' }}{\left|d^{\prime}\right|^{2} \operatorname{Imd}}} \quad \alpha=\frac{1+2 \operatorname{Red}+|\alpha|^{2}}{\operatorname{Imd}}
\end{array}\right.
$$

et dans $\mathrm{E}_{2}$ :

$$
\left\{s^{\prime}=s^{\frac{\alpha}{\alpha^{\prime}}} r^{\frac{\operatorname{Im} d^{\prime}}{\operatorname{Imd}}} \quad s^{\frac{\alpha}{\alpha^{\prime}}-\frac{\operatorname{Imd} d^{\prime}}{\operatorname{Imd}}}\right.
$$

On vérifie que l'application ainsi définie se prolonge bien en un homéomorphisme de $M_{1}$ dans elle inême, et le problème est résolu.

\section{Cas général}

- Lorsque l'on reste dans le cas des variétés correspondant à 
$\alpha\left\{\begin{array}{l}a \neq 0 \quad b \neq 0 \text { de la proposition 2, on procède comme précédemment, c'est-à-dire } \\ \text { et } a \neq b\end{array}\right.$ qu'on modifie la correspondance des points des trajectoires de $W$ et de $W^{\prime}$ de telle façon que l'application ainsi construite se prolonge en un homéomorphisme de la variété considérée sur elle-même. Pour ce faire, on est amené à considérer comme précédemment l'ensemble $\mathrm{E}$, et à définir la correspondance dans des parties de $\mathrm{E}$ situées entre des courbes du type $r=s^{P / q} P / q \in Q^{+}$,

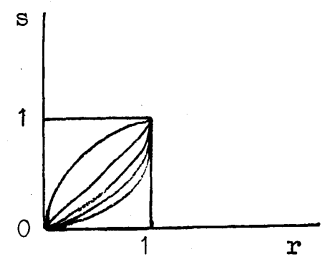

$$
E=\{(x, y) \mid r \leqslant 1 \text { et } s \leqslant 1\}
$$

Le nombre de courbes considérées correspondant au nombre d'éclatements successifs opérés. Les conclusions précédentes restent vraies.

- Il reste à traiter les cas $\alpha$ a $=0$ ou $b=0, \alpha$ a $=b$, $\beta$ et $Y$ mais dans ces cas le problème est plus simple car il s'agit de cas dégénérés et l'on peut même construire un automorphisme de $M$ qui envoie les trajectoires de $V$ sur celles de $V^{\prime}$. D'où :

4. THEOREME - Sur une surface algébrique complexe obtenue par éclatements successifs de $\mathbb{P}_{1}(\mathbb{C}) \times \mathbb{P}_{1}(\mathbb{C})$, l'ensemble des champs de vecteurs structurellement stables est un ouvert partout dense de I'ensemble des champs de vecteurs holomorphes.

5. Remarques

On fait une démonstration analogue à partir de $\mathbb{P}_{2}(\mathbb{C})$ ce qui généralise les résultats obtenus par J. Guckenheimer dans un article à parâtre.

\section{BIBLIOGRA PHIE}

[1] CARRELL ( $\left.J_{\bullet}\right)$, HOWARD (A.) and KOSNIOWSKI (c.) - Holomorphic Vector Fields on complex Surfaces (à parâtre).

[2] MATSUSHIMA (Y.) - Holomorphic vector fields on compact Kăhler manifolds, A.M.S., 1971 .

(Texte reçu le $8 / \mathrm{VI} / 1972$ )

Département de Mathématiques Université de Metz

Ile de Saulcy

57 - METZ 